\title{
Belastungsmatrix und „Masterplan 50plus“ der EDG Entsorgung Dortmund GmbH
}

\author{
T. Wulf
}

\section{Die EDG Entsorgung Dortmund GmbH und ihre demografischen Herausforderungen}

Die EDG Entsorgung Dortmund GmbH (EDG) ist ein 1990 gegründeter, kommunaler Entsorgungsfachbetrieb, welcher sich mit der Sammlung, dem Transport und der Verwertung von Abfällen und Wertstoffen beschäftigt. Zu den Kernaufgaben des Unternehmens zählen unter anderem die Abfallwirtschaftslogistik, die Reinigung der Straßen inklusive des Winterdienstes, die Pflege des Straßenbegleitgrüns sowie die Reinigung auf Gehwegen und der Betrieb von sechs Recyclinghöfen im Dortmunder Stadtgebiet. Damit ist das Leistungsspektrum an körperlicher Arbeit breit gefächert und reicht von organisatorisch planerischen Bürotätigkeiten bis hin zu körperlich äußerst fordernden Tätigkeiten. Um die rund 970 Beschäftigten der EDG möglichst lange erwerbsfähig zu halten und belastungsgerecht zu beschäftigen, befasst sich die EDG seit 2012 mit dem Thema Belastungsanalyse. Die Belastungsanalyse zielt dabei auf eine Analyse der Belastungs- und Gefährdungssituation von Mitarbeitenden, um deren Gesundheit und Sicherheit langfristig zu gewährleisten.

Ausschlaggebend für die Beschäftigung der EDG mit dem Thema Belastungsanalyse war unter anderem die demografische Situation in Deutschland. Folglich werden die zurzeit noch beschäftigten Personen der geburtenstarken Jahrgänge zunehmend altersbedingt aus dem Erwerbsleben ausscheiden. Dies ist eine Entwicklung, die auch die EDG in mannigfaltiger Weise betrifft. Im Folgenden werden daher die Auswirkungen des demografischen Wandels für die EDG aus zwei Perspektiven beleuchtet. Zum einen die Auswirkungen der Demografie nach innen, gemeint sind Auswirkungen auf die Belegschaft der EDG, und zum anderen die Auswirkungen der Demografie nach außen, womit die strukturellen Gegebenheiten der Gesellschaft und der Kundschaft der EDG gemeint sind. 


\subsection{Demografie nach innen: ältere Belegschaft}

Seit Gründung der EDG ist die Fluktuationsquote, insbesondere bei gewerblich-technischen Beschäftigten, welche mit hohen körperlichen Anforderungen konfrontiert sind und den Großteil der Beschäftigten darstellen, gering. Folglich altert die bei der Gründung durchschnittlich jüngere Belegschaft und es resultiert eine aktuell überdurchschnittlich hohe Altersstruktur. 2012 waren 42,6\% der EDG-Belegschaft 50 Jahre und älter. Im Jahr 2020 ist dieser Teil der Belegschaft auf 49,0\% angestiegen. Eine interne Vorausberechnung hat ergeben, dass bis zum Jahr 2025 ca. $20 \%$ der Beschäftigten und von 2026 bis 2035 nochmals ca. $50 \%$ der Belegschaft altersbedingt ausscheiden werden. Durch das ansteigende Durchschnittsalter sind im operativ-gewerblichen Bereich bei den schweren, körperlich auszuübenden Tätigkeiten eine sinkende Leistungsfähigkeit und steigende Krankenquoten der Beschäftigten zu erwarten. Verschärft wird diese Situation durch die geringe zur Verfügung stehende Anzahl an Menschen im erwerbsfähigen Alter, die zunehmend zu einem Fachkräftemangel und folglich zu Schwierigkeiten im Recruiting und der adäquaten Personalbereitstellung führt. Diese zu erwartenden Entwicklungen lassen einen Rückgang der Produktivität wahrscheinlich werden und stellen eine erhebliche Herausforderung für die EDG dar.

\subsection{Demografie nach außen: wandelnde Anforderungen an die Beschäftigten}

Die veränderten Anforderungen an die Beschäftigten, resultierend aus den äußeren demografischen Herausforderungen, lassen sich grundsätzlich in zwei Bereiche unterteilen. Zum einen wandeln sich die Anforderungen an die Beschäftigten durch strukturelle Veränderungen auf der Kundenseite und zum anderen durch technische Neuerungen im Arbeitsablauf.

Der erste Bereich ist vor allem dadurch bedingt, dass eine alternde Gesellschaft grundsätzlich vor ähnlichen Herausforderungen der körperlichen Leistungsfähigkeit steht wie die Belegschaft. Kundinnen und Kunden werden älter und damit tendenziell weniger körperlich belastbar. Das Serviceangebot der EDG muss gesteigert werden, um einem erhöhten Unterstützungsbedarf bei der Nutzung der Dienstleitungen gerecht zu werden und eine gleichbleibende Qualität gewährleisten zu können. Beispiele dafür sind der Vollservice ${ }^{1}$ in den Bereichen der abfallwirtschaftlichen Logistik oder Hilfestellungen bei der Entsorgung auf den Recyclinghöfen.

Eine veränderte gesellschaftliche Struktur führt zu einem veränderten Verhalten der Menschen in der Gesellschaft und damit zu einer Verände- 
rung der Dienstleistungsnachfrage. Eine Folge der Mediterranisierung ist die Verlagerung des sozialen Lebens in den öffentlichen Raum. Neben der Ausübung der originären Servicetätigkeiten in der Öffentlichkeit steigen die Anforderungen an die Soft-Skills der gewerblichen Beschäftigten. Durch die häufigeren Kontakte mit Kundinnen und Kunden wird eine freundliche und adressatengerechte Ansprache zunehmend wichtiger, da diese maßgeblich zum Image und zur Wahrnehmung des Unternehmens in der Öffentlichkeit beiträgt. Diese Veränderung führt zu einer Erweiterung des Anforderungsprofils der Tätigkeiten im gewerblichen Bereich.

Der zweite Bereich umfasst die Anforderungsänderungen durch technische Entwicklungen. Der zunehmende Digitalisierungsgrad beim Ausüben und Dokumentieren der Tätigkeiten im gewerblich-technischen Bereich führt zum Fort- und Weiterbildungsdruck der Beschäftigten. Ein Beispiel hierfür ist die Einführung eines neuen Abfallwirtschaftssystems. Dadurch werden Dokumentation oder Rückmeldungen nicht mehr auf herkömmliche Weise handschriftlich nach Beendigung der Tagestour festgehalten und anschließend von einem Verwaltungsmitarbeitenden in der Einsatzleitung digitalisiert, sondern bereits im Entsorgungs- oder Reinigungsrevier über ein mobiles Endgerät erfasst. Die Umstellung auf das Arbeiten mit digitalen Medien stellt für langjährige gewerbliche Mitarbeitenden teilweise eine Herausforderung dar.

\subsection{Zusammenfassung der demografischen Ausgangssituation und der EDG- Lösungsansatz}

Die zu erwartende altersbedingt abnehmende Produktivität durch tendenziell sinkende Leistungsfähigkeit und vermehrte Fehlzeiten sowie die zunehmende Schwierigkeit, adäquat qualifizierte Fachkräfte auf dem Arbeitsmarkt zu gewinnen, steht den stetig steigenden Anforderungen an die Beschäftigten und dem erforderlichen erhöhten Dienstleistungsgrad entgegen. Ein Ansatz, diesen Entwicklungen entgegenzutreten, ist es, die vorhandenen Beschäftigten in ihren Arbeitsbereichen langfristig leistungsund arbeitsfähig zu halten. Das Thema Gesundheit gewinnt in diesem $\mathrm{Zu}$ sammenhang zunehmend an Bedeutung. Mit Nachdruck wurden im Interesse aller Mitarbeitenden bereits im Jahr 2012 Maßnahmen und Projekte initiiert sowie durchgeführt, die dem Schutz, der Förderung und dem Erhalt ihrer Gesundheit dienen. Ein Resultat daraus ist die Entstehung einer differenzierten unternehmensspezifischen Belastungsmatrix. 


\section{Belastungsanalyse und Erstellung einer unternehmensspezifischen Belastungsmatrix}

Um den genannten Anforderungen des demografischen Wandels und dem Ansatz, Beschäftigte in ihren Arbeitsbereichen langfristig leistungs- und arbeitsfähig zu halten, gerecht zu werden, wurde von Februar bis Juli 2013 in einer Kooperation zwischen der EDG und dem Institut für Abfall, Abwasser und Infrastruktur-Management $\mathrm{GmbH}$, Ahlen (INFA) mit einem interdisziplinären Team eine ganzheitliche Belastungsanalyse der bei der EDG vorhanden gewerblich-technischen Tätigkeitsbereiche erstellt. Das interne Projektteam setzte sich im Wesentlichen aus verschiedenen Spezialisten aus den Bereichen Betrieb, betriebliches Gesundheitsmanagement und Personal zusammen. Ergänzt wurde dieses durch direkt betroffene gewerbliche Mitarbeitende und den Betriebsrat. Zur Erhebung der Belastungen ist ein Verfahren angewandt worden, welches auf der Leitmerkmalmethode aufbaut und durch weitere Merkmale ergänzt wurde. So wurden für die Beurteilung drei objektive Bewertungsindizes gebildet. Diese beinhalten körperliche lastabhängige Belastung mit den Merkmalen Heben, Halten, Tragen, Ziehen und Schieben, weitere körperliche lastunabhängige Belastungen, u.a. mit den Items Wege ohne Last, Witterung, Lärm und Geruch, und als dritter Baustein nicht-physische Belastungen mit den Merkmalen Verantwortung, Konzentration und Arbeitszeit bzw. Überstunden. Zudem wurden die potenziellen Auswirkungen der Belastung auf unterschiedlichen Körperregionen, wie beispielsweise Beine oder Rücken, berücksichtigt und beurteilt. Entsprechend der Ergebnisse der Belastungsanalyse erfolgte abschließend eine Verdichtung der Ergebnisse in eine weniger komplexe Darstellung mit der Clusterung Arbeitsplätze mit geringer, leichter, erhöhter und hoher Belastung.

Durch diese differenzierte Erhebung konnte im Ergebnis eine betriebsspezifische Belastungsmatrix erstellt werden, aus der folgende Kernaussagen abzuleiten sind:

- höchste physische Belastung bei der Rest- und Bioabfallsammlung der Lader-Tätigkeit im Citybereich (vielfach Kellerreviere)

- geringere physische Belastung bei der Sperrmüllsammlung

- geringste physische Belastung für Fahrer im Zustelldienst und für Mitarbeitende der Recyclinghöfe, Großwohnanlagen, bei der Depotcontainer-Standplatzreinigung und den Einzelposten in der Innenstadt (Papierkorbleerung) 
- hohe nicht-physische Belastung für Fahrer der Müllabfuhr und StraBenreinigung in stark verdichteten Bereichen, wie z.B. Innenstadt, sehr hohe Belastung für Fahrer von Kleinkehrmaschinen

- deutlich geringere nicht-physische Belastung in den übrigen untersuchten Bereichen

- unterschiedliche Ausprägung der Körperregionen-Belastungen abhängig vom Einsatzbereich

Abbildung 1: Bewertung der physischen Belastung am Arbeitsplatz-Bereich Abfallsammlung - (EDG Entsorgung Dortmund GmbH)

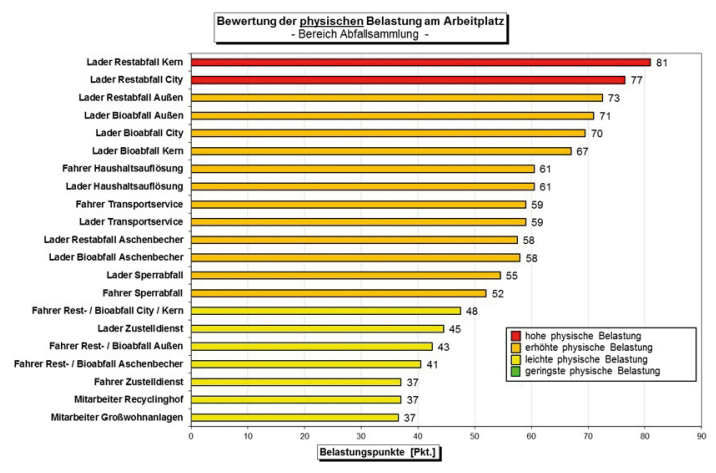

Abbildung 2: Bewertung der nicht-physischen Belastung am Arbeitsplatz-Bereich Abfallsammlung (EDG Entsorgung Dortmund GmbH)

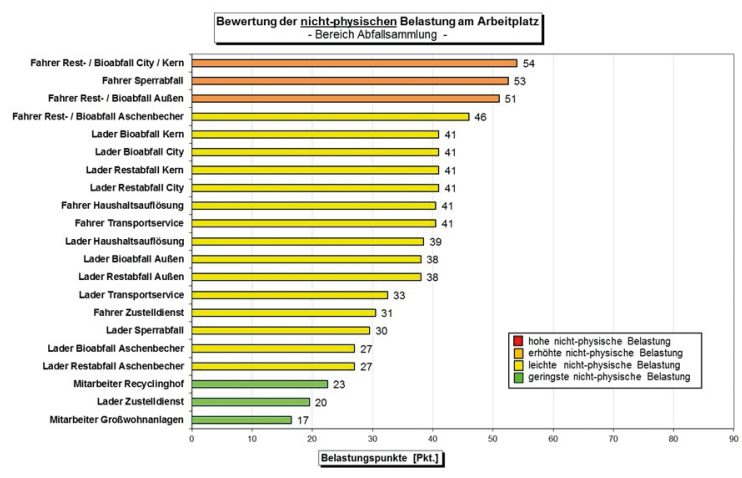




\section{Konzeptionierung praxistauglicher Maßnahmen}

Die differenzierten Ergebnisse der erstellten Belastungsmatrix bildeten die Grundlage für die Initiierung der nächste Projektphase, welche im Januar 2014 eingeleitet wurde: die Konzeptionierung praxistauglicher Maßnahmen, welche den Erhalt der Gesundheit und der Leistungsfähigkeit von den Beschäftigten sowie die Sicherung der Wettbewerbsfähigkeit gewährleisten sollen.

\subsection{Entwicklung und Strukturierung des Konzeptes der praktischen Umsetzung}

Bei der Entwicklung des Konzeptes standen folgende Leitfragen im Fokus und dienten als Orientierung:

- Wie kann eine individuelle, langfristige Einsatz-/Karriereplanung sichergestellt werden, die die Tätigkeitsprofile bzw. Arbeitsplatzanforderungen und die individuellen Mitarbeiterfähigkeiten abgleicht und überein bringt?

- Welche Methoden eignen sich, um die täglichen und langfristigen Belastungen der Mitarbeitenden zu reduzieren?

- Welche Maßnahmen der betrieblichen Gesundheitsförderung tragen zum Erhalt der Arbeitsfähigkeit bei?

- Welche technischen Hilfsmittel eignen sich, um die Belastungen am Arbeitsplatz zu reduzieren?

Aus den Leitfragen konnten schließlich interdisziplinäre Handlungsfelder für verschiedene Maßnahmen erschlossen werden. Diese umfassen die Arbeitsorganisation und Arbeitsplatzgestaltung, die Arbeitszeitstrukturierung, die Gestaltung der Lebensarbeitszeit und den Übergang ins Rentenalter sowie das präventive betriebliche Gesundheitsmanagement.

Die herausgearbeiteten Leitfragen und Handlungsfelder dienten als Basis für die finale Entwicklung praxistauglicher Maßnahmen, welche u.a. in der Konzeption des „Masterplan 50plus“ zusammengeführt werden.

\section{2 „Masterplan 50plus“-Maßnabmen in der betrieblichen Praxis}

Der „Masterplan 50plus“ umfasst die praktische Durchführung der konzeptionierten Maßnahmen zum langfristigen Erhalt der Leistungs- und Arbeitsfähigkeit der Beschäftigten im Betrieb, die aus der unternehmensspe- 
zifischen Belastungsmatrix abgeleitet wurden. Die Namensgebung „50plus" limitiert die Maßnahmen selbstverständlich nicht auf die Beschäftigtengruppe ab einem Alter von 50 Jahren, sondern verdeutlicht vielmehr, dass die Beschäftigten auch im Alter über 50 in der Lage sein sollen, ihre belastenden Tätigkeiten, ggf. in Abwandlung, auszuführen und somit einer beruflichen Perspektive im Unternehmen entgegensehen können.

Die folgend beispielhaft aufgeführten Maßnahmen des „Masterplan 50plus" wirken entlastend für die Beschäftigten und werden sukzessive realisiert:

- Optimierte Ausnutzung der täglichen Arbeitszeit mit begründet gelegten Pausen

- Rotation innerhalb der Teams: Wechsel von Fahrer- und Lader-Tätigkeit oder rotierende Lader-Tätigkeiten von Rausstellen, Laden und Reinstellen der Abfallsammelbehälter

- Gezielte Bildung von altersgemischten Teams

- Optimierungen bei der Arbeitsplatzgestaltung, bezogen auf die Behältergrößen: der Austausch von 240-Liter-Abfallsammelbehälter gegen leichtere 120-Liter-Gefäße sowie die Anpassung der Behälterausstattung, z.B. durch leichtgängige Räder

- Einsatz von technischen Hilfsmitteln, wie Kipp-, Zieh- oder Tragehilfen

Die genannten Maßnahmen, die mit deutlichen Auswirkungen auf die Arbeitsorganisation und -abläufe verbunden sind und mit langjährigen Gewohnheiten brechen, werden von ausführlichen Informationen für die betroffenen Beschäftigten begleitet, da dauerhaft nur deren Akzeptanz und Umsetzung den Erfolg gewährleisten. Gleichzeitig wurde der Kundenservice dahingehend geschult, die Maßnahmen ggf. auch gegenüber Kundinnen und Kunden zu kommunizieren.

Eine besondere Bedeutung innerhalb des „Masterplan 50plus“ kommt der Umsetzung des innerbetrieblichen Bereichs- und Abteilungswechsels zu. Vereinfacht dargestellt wechseln hier Beschäftigte dauerhaft aus dem vorherigen Arbeitsbereich, der auch im Alter noch mit harter, einseitiger körperlicher Belastung verbunden wäre, auf eine andere belastungsärmere Tätigkeit, die ohne oder nur mit geringen krankheitsbedingten Ausfallzeiten bis zum Renteneintrittsalter verrichtet werden kann. Anders als bisher, wechseln Beschäftigte z.B. von der Abfallwirtschaftslogistik zur Straßenreinigung oder von der Kehrtätigkeit in der Straßenreinigung zur Papierkorbleerung nicht erst bei eingetretener Leistungswandlung oder gesundheitlichen Einschränkungen, sondern präventiv, um diese erst gar nicht eintreten zu lassen. 
Ab dem Jahr 2015 wurden zwei entscheidende Meilensteine bei der Realisierung dieser Maßnahmen erreicht. Zum einen wurde eine Personaleinsatz-Datenbank in Betrieb genommen, aus der die Informationen

- des personenbezogenen Wechselbedarfs aufgrund von Krankheit oder gesundheitlichen Beeinträchtigungen,

- des Wechselwunsches,

- für den Wechsel notwendigen Qualifikationen, wie z. B. Führerschein der Klasse C/CE,

- der Empfehlungen des arbeitsmedizinischen Dienstes bzw. der Betrieblichen Gesundheitsförderung und

- der Priorität des Handlungsbedarfs

ersichtlich sind.

Zum anderen wurden im darauffolgenden Jahr die Vereinbarungen fixiert, die finanzielle Ausgleiche schaffen, wenn Mitarbeitende auf eine leistungsgewandelte und in der Regel geringer bezahlte Stelle wechseln. Damit ist eine große Hürde bei den Beschäftigten genommen, die aus finanziellen Gründen auch mit größeren gesundheitlichen Problemen nicht wechseln wollten oder konnten. An dieser Stelle sei angemerkt, dass Studien zur betrieblichen Gesundheitsvorsorge belegen, dass sich jeder investierte Euro langfristig mit dem Faktor fünf verzinst. ${ }^{2}$

\section{Fazit und Ausblick}

Mit der differenzierten Erhebung und Analyse der Belastungen der betrieblichen Tätigkeiten konnte eine aussagekräftige Belastungsmatrix erstellt werden. In Kombination mit der betrieblichen Datenbank liegt der EDG ein praxistaugliches und -erprobtes Instrument vor, mit dem eine individuell auf die Beschäftigten zugeschnittene Umsetzung realisiert werden kann. Dadurch profitieren einerseits die Beschäftigten, da sie langfristig leistungs- sowie arbeitsfähig bleiben, und so ein gesicherter Erwerbslebenslauf bis zum Renteneintrittsalter ermöglicht wird. Andererseits sichert diese langfristige Perspektive der Mitarbeitenden dem Unternehmen auch bei einer zunehmend alternden Belegschaft die Wettbewerbsfähigkeit. Die eingangs skizzierte erwarte demografische Entwicklung lässt dieses Instrument auch in Zukunft weiterhin an Wichtigkeit gewinnen.

Die Thematik des demografischen Wandels ist vielschichtig, komplex und vor allem individuell. Dies macht deutlich, dass sich verschiedene Handlungsfelder zwangsläufig überschneiden und die Bewältigung nicht nur über die Belastungsmatrix sowie den "Masterplan 50plus“ erreicht 
werden kann. Es ist unabdingbar, dass die Beschäftigten selbst, im Sinne ihrer eigenen Gesundheit, Präventionsmaßnahmen ergreifen. Die verschiedenen Unterstützungsangebote zur Gesundheitsprävention der EDG sind umfangreich und tragen im erheblichen Maße dazu bei, dass ihre Beschäftigten dieses Ziel erreichen.

Um langfristig leistungs- und wettbewerbsfähig zu blieben ist die stetige (Weiter-)Entwicklung sowie Durchführung neuer und effektiver Instrumente notwendig. Natürlicherweise sind aber auch bei einem wirtschaftlichen Unternehmen die Kapazitäten nicht unendlich. Daher wird neben den vorhandenen umfangreichen Maßnahmen kontinuierlich an weiterreichenden Lösungsansätzen gearbeitet. Mit Fokus auf innovative Ideen und um - nachrangig - kostendämpfende Effekte zu erzielen, beteiligt sich die EDG in unregelmäßigen Abständen an innovativen Projekten, wie beispielsweise von 2016 bis 2019 an dem vom Bundesministerium für Bildung und Forschung geförderten Forschungsprojekt „TErrA - Überbetriebliche Tätigkeitswechsel zum Erhalt der Arbeitsfähigkeit in regionalen Netzwerken"3, um weitere Beschäftigungsalternativen sowie gesundheitserhaltende Maßnahmen für die Beschäftigten zu evaluieren. Bei diesen Projekten neu gewonnene Erkenntnisse werden anschließend in die internen Prozesse eingebunden, um diese nachhaltig zu optimieren.

\section{Referenzen}

1 Vollservice ist ein Dienstleistungsangebot der EDG, bei dem im Rahmen des Leerungsvorgangs der Abfallsammelbehälter vom Standort bei Kundinnen und Kunden geholt und nach der Leerung am Müllsammelfahrzeug wieder an den Stellplatz zurückgestellt wird, ohne dass Kundinnen und Kunden das Gefäß an die Straße stellen müssen.

2 iga.report Wirksamkeit und Nutzen betrieblicher Prävention: https://www.iga-inf o.de/veroeffentlichungen/pressemitteilungen/wirksamkeit-bgf/.

3 Weitergehende Informationen zum Projekt finden sich unter https://taetigkeitsw echsel.de/. 
